\title{
The clonal growth behavior of melanocytes derived from melanocyte stem cells in glabrous skin
}

\begin{abstract}
Melanocyte stem cells (MSCs) play crucial roles in the pigmentation of human skin and hair, but methods to isolate and culture them are not well characterized. In our study, we cultured melanocytes derived from human fetal hair follicles and from glabrous adult foreskins. Three kinds of culture methods were employed, namely, via conventional enzyme digestion and cultivation, culture on a matrigel matrix and tissue culture. The results show that a special population of melanocytes occurred in primary cultures, which differed from mature epidermal melanocytes. Those cells displayed bipolar or tripolar phenotypes, proliferated very rapidly and their growth patterns resembled schools of fishes. Immunohistochemical staining with the HMB45 antibody confirmed that these cells were indeed melanocytes. We speculate that these melanocytes in clone-like growth patterns may have derived from MSCs or their progenies, which exist not only in the bulge region of hair follicles but also within the epidermis and/or dermis of glabrous skin.
\end{abstract}

Keywords: melanocyte stem cells, culture, hair follicles, glabrous skin
Volume I Issue I - 2017

Ru-zhi Zhang,' Qi Liu,' Sai Cheng, ${ }^{2}$ Jing-Zhu²
'Department of Dermatology, Suzhou University, China

${ }^{2}$ Department of Dermatology, Bengbu Medical College, China

Correspondence: Ru-zhi Zhang MD, Department of Dermatology, The Third Affiliated Hospital of Suzhou University, I85 Juqian Road, Changzhou 213003, China,

Email zhangruzhi628@163.com

Received: January 18, 2017| Published: April 13, 2017
Abbreviations: MSCs, melanocyte stem cells; HFs, hair follicles; UVR, ultraviolet radiation; DSCs, dermal stem cells; DMEM, dulbecco modified eagle medium; PBS, phosphate buffered saline; EDTA, ethylenediamine tetraacetic; ORS, outer root sheaths; FBS, fetal bovine serum

\section{Introduction}

Melanocytes (MCs) originate from neural crest cells and undergo a complex process of fate determination. They migrate through the dermis to the epidermis and to developing hair follicles (HFs) during embryonic development, are responsible for coloration of the skin and hair, and protect the epidermis from hazardous ultraviolet radiation (UVR). ${ }^{1}$ MCs in the skin are present both in the epidermal basal layer and in HFs. MCs in HFs are roughly divided into two distinct populations: mature differentiated MCs located in the hair bulbs, and melanocyte stem cells (MSCs) located in the bulge region of the $\mathrm{HF}^{2}{ }^{2}$ MSCs and/or their immediate progenies possess the characteristics of slow-cycling and self-renewing, and have the ability to supply amplifying cells to the hair matrix. They eventually differentiate into melanin-producing MCs during the anagen phase of the hair cycle and are responsible for hair color. ${ }^{3}$ Clinically, in vitiligo patients, skin repigmentation usually begins at the orifices of HFs, and then gradually expands fuses and covers the entire de-pigmented area. ${ }^{4}$ During that process, the progenies of MSCs migrate upward along the surface of the HF and proliferate to cause re-pigmentation of the skin.

MCs in HFs of mouse and human skin have been extensively studied, ${ }^{5,6}$ but not much is known about MSCs in glabrous skin, such as the foreskin. Recently, some studies have reported that multi potent dermal stem cells (DSCs) isolated from human neonatal foreskins that lack HFs are able to differentiate into multiple cell lineages, including MCs. Those studies showed that MCs derived from DSCs could produce pigment and express melanocytic markers, such as MITF, DCT, TYRP1, S100, HMB45 and E-cadherin. ${ }^{7}$ Those MCs acted in the same manner as mature epidermal MCs. In the present study, we used various culture methods and conditions to observe melanocyte growth, and found that some MCs grew in patterns resembling schools of fishes, suggesting the presence of MSCs and/or their progenies not only in HFs but also in glabrous skin.

\section{Materials and methods}

\section{Isolation and culture of cells from HFs}

Fetal scalp skin specimens were obtained within $2 \mathrm{~h}$ of death and written informed consent was obtained from each family. Specimens were immediately immersed in Dulbecco modified Eagle medium (DMEM) supplemented with 400U/ml penicillin and 400g/ $\mathrm{ml}$ streptomycin. The epidermis, the upper $1 \mathrm{~mm}$ of the dermis and subcutaneous adipose tissues were manually removed with a scalpel. The remaining tissue was cut into $0.5 \mathrm{~cm} \times 3.0 \mathrm{~cm}$ pieces and immersed in $0.25 \%$ collagenase type IV (Sigma, C5138) for $2-4 \mathrm{~h}$ at $37^{\circ} \mathrm{C}$. Many individual HFs in all stages of growth were then freed of dermal tissue by exhaustive washing in $0.01 \mathrm{M}$ phosphate buffered saline (PBS) and removal with tweezers. Washing was continued until individual free HFs appeared to be pure.

The freed HFs were then treated with $0.25 \%$ trypsin and $0.53 \mathrm{mM}$ ethylenediamine tetraacetic acid (EDTA) for $10 \mathrm{~min}$ at $37^{\circ} \mathrm{C}$ to release cells located in the outer root sheaths (ORS) of these HFs. The suspensions were filtered through a $200 \mathrm{~m}$ filter, and centrifuged at $1000 \mathrm{r} / \mathrm{min}$ for $5 \mathrm{~min}$. The obtained cell pellets were re-suspended in M254 medium supplemented with human melanocyte growth supplement (HMGS, Life Technologies, 1671858), seeded in 6-well plates at a density of $5 \times 105 \mathrm{cells} / \mathrm{ml}$ and then cultured in a humidified atmosphere containing $5 \% \mathrm{CO}_{2}$ at $37^{\circ} \mathrm{C}$.

\section{Immunofluorescence of HF MCs}

After 2-3 weeks in primary culture, the HF MCs reached approximately $80 \%$ confluence and were sub cultured by differential trypsinization. At the third passage, the cells were fixed with $4 \%$ 
paraformaldehyde for $15 \mathrm{~min}$ at room temperature, then incubated in $0.1 \%$ Triton X-100 in PBS for $10 \mathrm{~min}$. After two successive washes in PBS, cells were incubated with a blocking solution containing $5 \%$ BSA (Sigma) at room temperature for $60 \mathrm{~min}$. The mouse monoclonal antibody HMB-45 (DAKO $\square$ M063429-2) was used at a 1:100 dilution and was incubated overnight at $4^{\circ} \mathrm{C}$. Specifically bound HMB-45 antibody was detected with a goat anti-mouse Alexa Fluor 488-conjugated secondary antibody (1:500;ab150105) and was incubated for $1 \mathrm{~h}$ at room temperature in the dark. All antibodies were diluted as noted in PBS/0.1\% BSA solution and an isotype-matched mouse IgG was used as a control. Nuclei were counterstained with 4 , 6-diamidino-2-phenylindole (DAPI, Invitrogen) at a 1:1000 dilution. All images were taken with a fluorescence microscope (Olympus,).

\section{Cultivation of HF cells on matrigel}

Basement Membrane Matrix (Corning BioCoat $\square$ 354277) is composed of laminin, type IV collagen, nestin, heparin sulfate glycoprotein, etc. At room temperature, the matrix polymerizes to form a three-dimensional scaffold that simulates the in vivo cell basement membrane, and contributes to the cultivation, proliferation and differentiation of cells in vitro. Without a feeder layer, the matrix can provide an ideal substrate support for the growth of cells. For preparation of the three-dimensional model using matrigel, everything that contacts the matrigel should be precooled when coated on culture plates. Matrigel and DMEM medium were mixed at a ratio of $1: 2$ and were incubated at $37^{\circ} \mathrm{C}$ for $4 \mathrm{~h}$ to promote gel formation. The mixed cell suspension was seeded on the matrigel matrix, and cells were cultured with DMEM supplemented with $10 \%$ fetal bovine serum (FBS) and were observed by light microscopy every two days.

\section{Tissue culture of foreskins}

Foreskin specimens were obtained from routine circumcisions and were cleaned. After subcutaneous tissues were removed, the foreskin specimens were cut into $0.5 \mathrm{~m} \times 0.5 \mathrm{~cm}$ pieces and were divided into two parts: one part for tissue culture and the other part for the culture of epidermal MCs. For tissue culture, the processed tissue specimens were directly plated into wells of 6-well plates that had been precoated with FBS to promote tissue adherence. After incubation with $5 \% \mathrm{CO}_{2}$ at $37^{\circ} \mathrm{C}$ for $2 \mathrm{~h}, 500 \mu \mathrm{l}$ DMEM containing $10 \%$ (vol/vol) FBS were added into each well. After $12 \mathrm{~h}, 500 \mu \mathrm{l}$ media were added into each well. The media were replaced every 2-3 days.

\section{Culture of epidermal MCs from foreskins}

For cell culture, the processed tissue specimens, which only contained epidermis and dermis, were incubated in $2.4 \mathrm{U} / \mathrm{ml}$ dispase (Roche, Cat. No.4942078001) in PBS for approximately $1.5 \mathrm{~h}$. The epidermis was separated from the dermis using tweezers. The remainder of the processing program was identical to the treatment of free HFs noted above to free epidermal cells. The obtained cell suspensions were incubated with medium 254 and $\mathrm{HMGS}$ at $37^{\circ} \mathrm{C}$ in a humidified atmosphere containing $5 \% \mathrm{CO}_{2}$ and the medium was changed every 2 days. When the cells reached approximately $80 \%$ confluence, they were trypsinized and sub cultured. In the process of passage, keratinocytes in the cultures were removed by differential trypsinization and treatment with $100 \mu \mathrm{g} / \mathrm{ml}$ geneticin (G418 sulfate, BIOSHARP, 345810) was used to eliminate contaminating fibroblasts.

\section{Results}

\section{Isolation and culture of HF cells}

After the treatment with Collagenase type IV, the scalp skin was loose and it was easy to pull the HFs out using forceps. After digestion with trypsin/EDTA, individual cells were released from the free HFs. In primary cultures, 3 kinds of cells were observed, MCs, keratinocytes and a few fibroblasts. At passage 3, a relatively pure population of MCs was obtained following differential trypsinization to eliminate keratinocytes and treatment with genetic in to kill fibroblasts. Two types of MCs were observed: one type was unpigmented MCs with small bodies that were bipolar or in some cases tripolar, and these cells proliferated very quickly. The other type was intensely pigmented MCs with multiple dendrites; these cells did not proliferate and disappeared after the third passage (Figures 1A \& 1B).

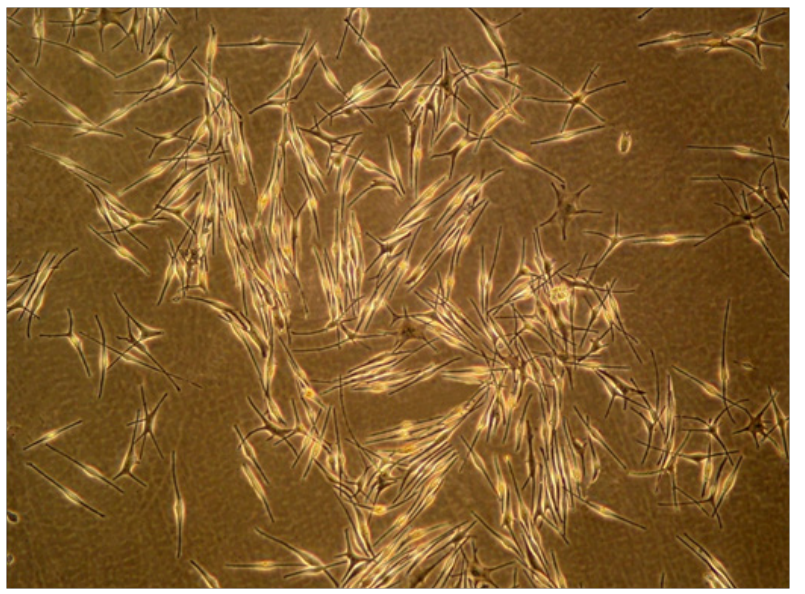

Figure 1 Cultivation of HF Mcs by differential enzyme digestion.

(A) Relative pure population of MCs at passage 3 included un-pigmented MCs with bipolar or in some cases tripolar, and pigmented MCs with multiple dendrites $(100 \times)$; (B) un-pigmented MCs had an appearance of small bodies and bipolar $(100 \times)$.

\section{Cultivation of HF cells on matrigel}

HF cells were cultured on matrigel with DMEM and FBS. After culture for 2 weeks, 3 kinds of cells were observed: a large number of keratinocytes identified by their cobblestone morphology, a few fibroblasts characterized by their generally elongated spindle-shaped morphology, and MCs, which were dendritic, bipolar or multipolar. Surprisingly, 2 types of MCs were observed: scattered MCs that had a multipolar dendritic appearance and were larger and intensely pigmented and MCs that grew out in groups, which resembled schools of fishes and showed a clonal growth behavior. The latter types of MCs were bipolar, had a good diopter and proliferated quickly (Figures 2A \& 2B).

\section{Immunofluorescence of HF MCs}

Immunohistochemical staining with the HMB-45 antibody was performed on the HF MCs at the third passage. The results showed that almost all cells were positive with intense green fluorescence, indicating the presence of moderate to high levels of the antigen defined by HMB-45, which is a specific marker for melanosomes. 
This staining pattern confirmed that these cells were indeed MCs. HMB-45-positive cells accounted for a large proportion of cells with smaller cell bodies, good diopters and bipolar shapes. On the tips of dendrites and at the periphery of the cell body, the green fluorescence was stronger, demonstrating the regions rich in melanosomes (Figure $3)$.

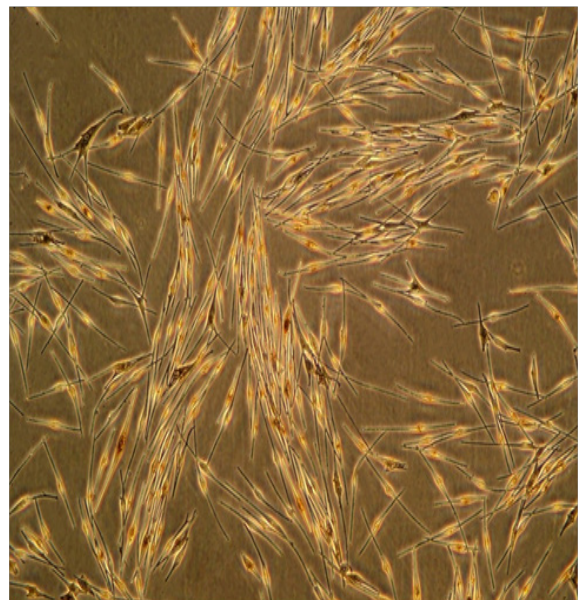

Figure 2 Cultivation of HF Cells on Matrigel.

(A) 3 kinds of cells were observed cultured on matrigel: keratinocytes identified by their cobblestone morphology, fibroblasts characterized by their elongated spindle-shaped morphology, and MCs were dendritic (100x); (B) groups of MCs were bipolar, a good diopter, which resembled schools of fishes (200x).



Figure 3 Immunohistochemical staining of Mcs.

Follicular MCs were stained with HMB-45 antibody. The results showed these cells were indeed melanocytes (I00X).

\section{Tissue culture of foreskins}

The foreskin tissues adhered to the culture plates within a few $\mathrm{h}$ after inoculation. By day 3, some keratinocytes showing a cobblestonelike appearance had grown out around the foreskin tissues. A few MCs with slender dendrites were scattered among the keratinocytes. By day 14 , some of the original tissues were floating and were removed by replacing the media. The migrated and adherent cells proliferated rapidly. Interestingly, besides the typical MCs, we observed another type of MCs, which were spindle-shaped, proliferated quickly, and grew in patterns resembling schools of fishes, among which a few were pigmented (Figures $4 \mathrm{~A} \& 4 \mathrm{~B}$ ).

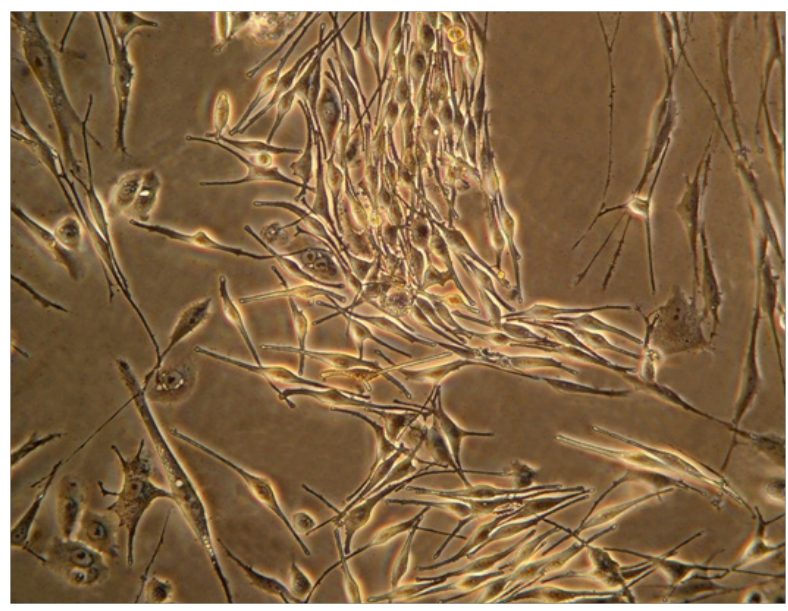

Figure 4 Tissue culture of foreskin cells.

$(A, B)$ In addition to keratinocytes and the typical MCs, a clonal growth behavior of MCs was observed, which present as spindle, proliferated quickly, grew in patterns resembling schools of fishes (100x).

\section{Culture of epidermal MCs from foreskins}

After sufficient enzyme digestion of foreskins, we separated the epidermis from the dermis as much as possible to reduce the subsequent fibroblast contamination. Epidermal cells from the foreskins were cultured in M254 medium supplemented with HMGS, which is suitable for melanocyte growth but not for other types of cells. In the primary cultures, the keratinocytes showed colony-like growth, and were arranged densely with a clear boundary. On the contrary, the MCs around those keratinocytes exhibited a parallel arrangement, and had elongated, bipolar or tripolar morphologies with varying degrees of pigmentation (Figure 5A).

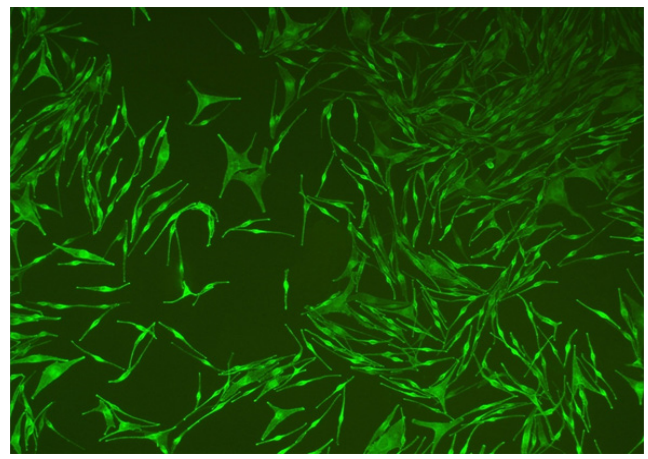

Figure 5 Cultivation of epidermal Mcs from foreskin.

(A) Before passaging of primary culture, keratinocytes and mealnocytes were both present and grown well. mealnocytes around keratinocytes displayed a parallel arrangement(I00x). (B) After passaging, epidermal MCs exhibited multiple dendrites and intense pigmention(I00x). (C,D) A group of melanocytes showed clonal growth patterns, bipolar, which differ from common epidermal melanocytes. Obviously, these clonal melanocytes originated from MSCs and/or their immediate progenies. (C:100x; D: 200x). 
After further passaging, relatively pure MCs were obtained by differential trypsinization. In most cases, human epidermal MCs had small bodies with a good diopter, and obviously multiple dendrites, however, they did not show clonal growth behavior (Figure 5B). Surprisingly, some groups of cells in one culture well were observed. At first glance, these cells looked like grains scattered in the culture plate because of their good refraction. A closer examination revealed many fusiform cells that grew in groups (Figures 5C \& 5D). These cells proliferated very rapidly and showed clonal growth patterns. They were obviously different from common epidermal MCs, and were classified as MSCs.

\section{Discussion}

According to the published literature, during embryonic development, melanocytic precursors, called melanoblasts, emerge from pluripotent cells, migrate from the neural crest and then colonize in the skin. ${ }^{8}$ The MSC system has many unique advantages not only for the study of mechanisms involved in pigmentary diseases but also to provide a sufficient supply of MCs for cell-based treatments. ${ }^{9}$ The maintenance and regeneration of the melanocyte population is dependent on MSCs that are located in the bulge and the sub-bulge areas of HF. ${ }^{2,10}$ Premature differentiation of bulge MSCs is also observed during physiological hair graying in mice and in humans. ${ }^{11}$ Amelanotic MCs (AMMCs) from HFs, which may be MSCs and/or their immediate progenies, were first successfully cultured by Tobin et al. ${ }^{12}$ and have been confirmed by other research teams. ${ }^{13,14}$

In the present study, we used two distinct methods to culture cells from HFs. In conventional monolayer culture using an M245 selective medium, the predominant cells were AMMCs that were characterized as having small bodies, bipolar shapes and were rapidly proliferating. ${ }^{14}$ Staining with the HMB-45 antibody confirmed that they were indeed melanocytes. It is known that HMB-45 specifically recognizes the melanosomal protein gp100, also known as Pmel17, and is a melanocyte lineage-specific marker. The few large and pigmented MCs did not proliferate and disappeared after the third passage. When HF cells were cultured on matrigel, groups of AMMCs were observed in the primary cultures.

MSCs have also been demonstrated to exist in the dermis other than the bulge region of HFs. ${ }^{15-17}$ That evidence comes from clinical studies of patients with vitiligo, who were given a series of treatments to stimulate re-pigmentation of the skin. ${ }^{18}$ After those treatments, some tyrosinase-positive MCs were detected along the basement membrane in the previously de-pigmented palm skin. This clinical evidence supports the existence of a MSC reservoir in extra follicular skin. ${ }^{16}$ Some authors have hypothesized that human extra follicular skin contains a stem cell population, called human DSCs, which are capable of differentiating into a wide variety of cell types, such as MCs. ${ }^{17,19}$ It has been suggested that DSCs may be a source of extra follicular epidermal MCs. ${ }^{20}$

In general, epidermal MCs are mature cells, showing multiple dendrites and pigmentation. However, we found a special type of melanocyte during the process of cultivating foreskin tissue and conventional MCs. Those cells have a high proliferative capacity, show a clonal growth behavior, and are arranged densely, resembling a school of fishes. ${ }^{13}$ Their morphology and characteristics are similar to AMMCs from HFs, but were more immature. In theory, there is no HFs in the foreskin, so these cells should not have migrated from the bulge region of HFs. We suspect that foreskin epidermal MCs may be derived from MSCs, which may be present in the epidermis or dermis. ${ }^{17,19}$

In summary, using two different sources of skin and different culture methods, we obtained a pure population of MCs, which displayed a clonal growth behavior, proliferated very quickly, and were present as fusiform or bipolar or tripolar shapes. These results suggest that MSCs and/or their immediate progenies, such as AMMCs, exist not only in the bulge region of HFs but also within the epidermis or dermis of glabrous skin. ${ }^{17-20}$ These MSCs provide a new basis to understand the pathological changes caused by defects or dysfunction in stem cells, such as hair graying, vitiligo and melanoma. ${ }^{10,15,19}$

\section{Acknowledgements}

The authors are very grateful to Professor V. J. Hearing, Laboratory of Cell Biology, National Cancer Institute, Bethesda, MD, USA, for English-language element of this paper. The work was supported by the National Natural Science Foundation of China (No 81673078) and the Clinical Medical Science and Technology Special Fundation of Jiangsu Province (BL 2014036).

\section{Conflict of interest}

The author declares no conflict of interest.

\section{References}

1. Blanpain C, Sotiropoulou PA. A dominant role of the hair follicle stem cell niche in regulating melanocyte stemness. Cell Stem Cell. 2010;6(2):95-96.

2. Buac K, Pavan WJ. Stem cells of the melanocyte lineage. Cancer Biomark. 2007;3(4-5):203-209.

3. Nishimura EK. Melanocyte stem cells: a melanocyte reservoir in hair follicles for hair and skin pigmentation. Pigment Cell Melanoma Res. 2011;24(3):401-410.

4. Yamaguchi Y, Hearing VJ. Melanocytes and their diseases. Cold Spring Harb Perspect Med. 2014;4(5) pii: a017046.

5. Godwin LS, Castle JT, Kohli J S, et al. Isolation, culture, and transfection of melanocytes. Curr Protoc Cell Biol. 2014;63(1-8):1-20.

6. Hirobe T. How are proliferation and differentiation of melanocytes regulated? Pigment Cell Melanoma Res. 2011;24(3):462-478.

7. Li L, Fukunaga-Kalabis M, Herlyn M. Isolation and cultivation of dermal stem cells that differentiate into functional epidermal melanocytes. Methods Mol Biol. 2012;806:15-29.

8. Nishikawa-Torikai S, Osawa M, Nishikawa S. Functional characterization of melanocyte stem cells in hair follicles. $J$ Invest Dermatol. 2011;131(12):2358-2367.

9. Lang D, Mascarenhas JB, Shea CR. Melanocytes, melanocyte stem cells, and melanoma stem cells. Clin Dermatol. 2013;31(2):166-178.

10. Lee JH, Fisher DE. Melanocyte stem cells as potential therapeutics in skin disorders. Expert Opin Biol Ther. 2014;14(11):1569-1579.

11. Endou M, Aoki H, Kobayashi T, et al. Prevention of hair graying by factors that promote the growth and differentiation of melanocytes. J Dermatol. 2014;41(8):716-723.

12. Tobin DJ, Colen SR, Bystryn JC. Isolation and long-term culture of human hair-follicle melanocytes. J Invest Dermatol. 1995;104(1):86-89.

13. Zhang RZ, Zhu WY, Li HZ, et al. Culture of amelanotic melanocytes derived from human fetal hair follicles. In Vitro Cell Dev Biol Anim. 2013;49(9):689-694. 
14. Na GY, Paek SH, Park BC, et al. Isolation and characterization of outer root sheath melanocytes of human hair follicles. $\mathrm{Br} J$ Dermatol. 2006;155(5):902-909.

15. Seleit I, Bakry OA, Abdou AG, et al. Immunohistochemical study of melanocyte-melanocyte stem cell lineage in vitiligo; a clue to interfollicular melanocyte stem cell reservoir. Ultrastruct Pathol. 2014;38(3):186-198.

16. Glover JD, Knolle S, Wells KL, et al. Maintenance of distinct melanocyte populations in the interfollicular epidermis. Pigment Cell Melanoma Res. $2015 ; 28(4): 476-480$.

17. Zabierowski SE, Fukunaga-Kalabis M, Li L, et al. Dermis-derived stem cells: a source of epidermal melanocytes and melanoma? Pigment Cell Melanoma Res. 2011;24(3):422-429.
18. Sisti A, Sisti G, Oranges CM. Effectiveness and safety of topical tacrolimus monotherapy for repigmentation in vitiligo: a comprehensive literature review. An Bras Dermatol. 2016;91(2):187-195.

19. Hoerter JD, Bradley P, Casillas A, et al. Extrafollicular dermal melanocyte stem cells and melanoma. Stem Cells Int. 2012;2012:407079.

20. Li L, Fukunaga-Kalabis M, Yu H, et al. Human dermal stem cells differentiate into functional epidermal melanocytes. J Cell Sci. 2010;123(Pt 6):853-860 